\title{
Programa de juegos de razonamiento lógico para estimular las operaciones concretas en niños de Huancayo
}

\section{Program of logical reasoning games to stimulate the concrete operations in children from Huancayo}

\section{RESUMEN}

Objetivos: Determinar los efectos de la aplicación de un programa de juegos de razonamiento lógico para estimular las operaciones concretas en niños de segundo grado de educación primaria que asistían a un consultorio psicológico privado de la ciudad de Huancayo. Métodos: Se realizó la aplicación de actividades significativas de enseñanza aprendizaje, sistemáticamente organizadas, que consideraba las siguientes dimensiones: juegos de seriación, juegos de clasificación y juegos de conservación. Durante 22 sesiones realizadas tres veces por semana. Para determinar el dominio de las destrezas, se recurrió a la batería psicopedagógica "Forcab". Para probar que el programa de juegos incrementaba o no las operaciones concretas en los niños se utilizó la prueba de Wilcoxon para muestras relacionadas, prueba paramétrica equivalente a la " $t$ " student, pudiéndose concluir que los juegos de seriación, los de clasificación y los de conservación ayudaron a incrementar las operaciones concretas de los niños. Resultados: Existen diferencias estadísticamente significativas entre los puntajes de la evaluación pre y post experimental, con lo cual se demostró que sí es posible mejorar los procesos cognoscitivos en los niños de 7 y 8 años de edad. Conclusiones: Se demuestra que sí es posible mejorar los procesos cognoscitivos en los niños de 7 y 8 años de edad. La aplicación del programa experimental de estimulación de los procesos cognoscitivos mejoró significativamente el desarrollo de las operaciones concretas en los niños. Sí es posible diseñar un programa para mejorar un estadio del desarrollo intelectual, como son las operaciones concretas, a través de su estimulación.

Palabras clave: Seriación, clasificación, noción, conservación.

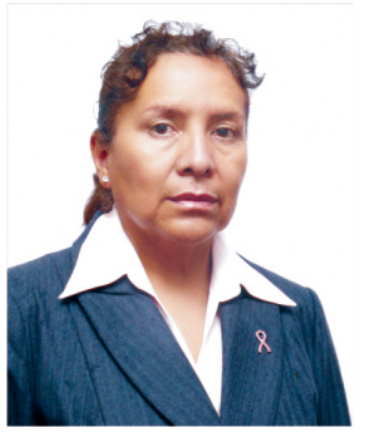

Carmen Aliaga Arroyo Asociación Psicológica Activa

\section{ABSTRACT}

Objectives: To determinate the effects of the application of logical reasoning games program to stimulate the concrete operations in children of second grade in primary education that they were attended at the psychological private consulting room in Huancayo city. Methods: It has been done the application of significant activities of teaching and learning, systematically organized; which considered the following dimensions: seriation, classification and conservation games. During 22 sessions were done three times per week. To determine the domain of the dexterities, it was appealed to the psicopedagogic battery "Forcab". To prove that the program of games improve or not the concrete operations in children was

1 Mg. en Psicología, Presidenta de la Asociación Psicológica Activa (ASPAC). Couch internacional de la Academia de Couching y Liderazgo Amerilideres (ACCA) 
used the Wilcoxon test for related samples, parametric test equivalent to the "t" student, being able to conclude that the seriation, classification and conservation games helped to increase the concrete operations of children. Results: Differences statistically significant exist among the scores of the evaluation pre and post experimental, which was demonstrated that it is possible improve the cognitive processes in children of 7 and 8 years old. Conclusions: It is demonstrated that it is possible improve the cognitive processes in children of 7 and 8 years old. The application of the experimental program of stimulation in the cognitive processes improved the develop of concrete operations significantly in children. Also, it is possible design a program to improve part of the intellectual development like they are the concrete operations, through their stimulation.

Key words: Seriation, classification, notion, conservation.

\section{INTRODUCCIÓN}

Según los investigadores: Majluf (1), Frisancho (2), Meza e Inga (3), Carrasco (4), Macías (5), Tejeda (6), Ruesga (7) y Ruiz (8); las nociones lógico matemáticas que adquieren los niños en sus primeros grados constituyen la base de todo su aprendizaje lógico matemático futuro. El desarrollo cognitivo, como afirma Majluf (1), Ruíz (8) "no es un proceso que ocurre en forma necesaria y automática conforme el sujeto va creciendo". Por lo que es necesaria su estimulación y, en este sentido, los juegos de razonamiento lógico constituyen un medio eficaz para el desarrollo de las capacidades cognitivas del niño, relacionadas con operaciones mentales propias de la matemática. Además, favorecen la construcción de esquemas mentales que permiten la asimilación activa de la lectura y como afirma Condemarín (9), "mientras más se favorezca la construcción de las nociones lógico matemáticas, más se mejoran la motivación y la calidad del aprendizaje de las matemáticas, disminuyendo así el tradicional temor a esta disciplina".

Esta investigación dio respuesta a la siguiente interrogante $\dot{Q}$ ué efectos tiene un programa de juegos de razonamiento lógico para estimular las operaciones concretas?, en este sentido, se determinó los efectos de la aplicación de un programa de juegos de razonamiento lógico en una muestra de estudiantes que asistían a un consultorio psicológico privado de la ciudad de Huancayo.

La hipótesis sostenía que la aplicación del programa mejoraría significativamente la etapa del desarrollo de las operaciones concretas de los niños.

Determinar los efectos de la aplicación de un programa de juegos de razonamiento lógico para estimular las operaciones concretas en niños de segundo grado de educación primaria que asistían a un consultorio psicológico privado de la ciudad de Huancayo.

\section{MATERIAL Y MÉTODOS}

La aplicación del programa de juegos de razonamiento lógico se dio en el consultorio psicológico privado de la investigadora a través de la aplicación de actividades significativas de enseñanza aprendizaje, sistemáticamente organizadas, que consideraba las siguientes dimensiones: juegos de seriación (cuyo indicador era ordenar correctamente los elementos en una serie, teniendo en cuenta que cada uno de ellos es simultáneamente el mayor de los que le anteceden y el menor de los que le suceden o viceversa); juegos de clasificación (cuyo indicador era clasificar elementos según características diversas como color, forma, tamaño y grosor), juegos de conservación (cuyo indicador era enunciar propiedades invariantes del objeto, aún cuando perceptualmente éste cambie de forma). Durante 22 sesiones realizadas tres veces por semana. Se trabajó de 30' a 40' por sesión, de manera personalizada. Se tomó en cuenta como variable independiente el programa de juegos de razonamiento lógico, como variable dependiente el desarrollo de las operaciones concretas, siendo las variables intervinientes controladas, la edad niños de 7 y 8 años, sexo masculino y femenino, escolaridad segundo de primaria $y$ nivel socioeconómico medio. 
Tabla 1: Operacionalización de variable independiente

\begin{tabular}{|c|c|c|c|}
\hline & Dimensiones & Indicadores & Escala de medición \\
\hline$\frac{O}{U}$ & $\begin{array}{l}\text { Programa de } \\
\text { juegos de } \\
\text { seriación }\end{array}$ & $\begin{array}{l}\text { Ordena correctamente los elementos en una serie, teniendo en } \\
\text { cuenta que cada uno de ellos es simultáneamente el mayor de } \\
\text { los que le anteceden y el menor de los que le suceden o } \\
\text { viceversa. }\end{array}$ & Nominal \\
\hline 㟧邑 & $\begin{array}{l}\text { Programa de } \\
\text { juegos de } \\
\text { clasificación }\end{array}$ & $\begin{array}{l}\text { Clasifica elementos según características diversas: color, forma, } \\
\text { tamaño, grosor.. }\end{array}$ & Nominal \\
\hline$\frac{\alpha}{\alpha}$ & $\begin{array}{l}\text { Programa de } \\
\text { juegos de } \\
\text { conservación }\end{array}$ & $\begin{array}{l}\text { Enuncia propiedades invariantes del objeto, aún cuando } \\
\text { perceptualmente éste cambie de forma. }\end{array}$ & Nominal \\
\hline
\end{tabular}

Tabla 2: Operacionalización de variable dependiente

\begin{tabular}{|c|c|c|c|}
\hline & Dimensiones & Indicadores & Escala de medición \\
\hline \multirow{3}{*}{ 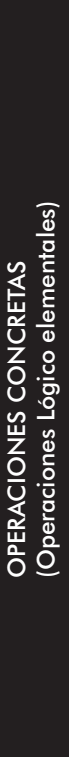 } & Seriación & $\begin{array}{l}\text { A. Al realizar seriaciones comprende las operaciones de } \\
\text { Transitividady Reversibilidad. } \\
\text { a. Operación de Transitividad } \\
\text { - Compara tres elementos. } \\
\text { - Deduce que si A es mayor que B y B es mayor que C, } \\
\text { entonces A es mayor que C. } \\
\text { b. Operación de reversibilidad } \\
\text { - Realiza simultáneamente dos seriaciones inversas, } \\
\text { - ya sea en forma creciente o decreciente. } \\
\text { - Ordena el elemento más pequeño (o el más } \\
\text { grande) del conjunto que se va a seriar, y el más } \\
\text { grande de los ya ordenados (o el más pequeño). }\end{array}$ & Ordinal \\
\hline & Clasificación & $\begin{array}{l}\text { A. Al realizar clasificaciones, domina la noción de } \\
\text { inclusión. } \\
\text { - Forma subclases, que luego incluye en una clase de } \\
\text { mayor extensión. } \\
\text { - Establece una relación entre el todo y la o las partes. } \\
\text { - Forma clases y subclases. }\end{array}$ & Ordinal \\
\hline & $\begin{array}{l}\text { Noción de } \\
\text { Conservación }\end{array}$ & $\begin{array}{l}\text { A. Al realizar juegos de conservación, enuncia propiedades } \\
\text { invariantes del objeto, aún cuando perceptualmente } \\
\text { éste cambie de forma. } \\
\text { B. Reversibilidad, Se imagina en forma coordinada el } \\
\text { conjunto de acciones realizadas y su regreso a su punto de } \\
\text { partida. }\end{array}$ & Ordinal \\
\hline
\end{tabular}


Tabla 3: Operacionalización de variables intervinientes

\begin{tabular}{|c|c|c|c|}
\hline Variable controlada & Dimensiones & Indicadores & Escala de medición \\
\hline Edad Cronológica & 7,8 años & Se encuentra entre 7 y 8 años & Ordinal \\
\hline \multirow{2}{*}{ Sexo } & Masculino & Apariencia física masculino & Nominal \\
\hline & Femenino & Apariencia física femenina & Nominal \\
\hline Escolaridad & $2^{\circ}$ primaria & $\begin{array}{l}\text { Alumnos matriculados y que } \\
\text { siguen estudios en } 2^{\circ} \text { de primaria }\end{array}$ & Ordinal \\
\hline $\begin{array}{l}\text { Nivel } \\
\text { socioeconómico }\end{array}$ & Medio & $\begin{array}{l}\text { Asisten a un Centro Educativo } \\
\text { Particular }\end{array}$ & Nominal \\
\hline
\end{tabular}

\section{RESULTADOS}

Se encontró que existían diferencias estadísticamente significativas entre los puntajes de la evaluación pre y post experimental, con lo cual se demostró que sí es posible mejorar los procesos cognoscitivos en los niños de 7 y 8 años de edad. Pudiéndose asumir que la aplicación del programa experimental de estimulación de los procesos cognoscitivos mejoró significativamente el desarrollo de las operaciones concretas en los niños.
Como puede verse en la tabla 4, el número de elementos para los cuales el valor de la variable post-test seriación es considerablemente mayor que el de la variable pre-test seriación; por tanto, se rechaza la hipótesis nula de que la valoración de pre-test seriación es igual que la del post-test seriación para $5 \%$ de nivel de significación. Por lo tanto se puede concluir que los juegos de seriación, ayudan a incrementar las operaciones concretas de los niños.

Tabla 4: Análisis de cambios significativos en los juegos de seriación

\begin{tabular}{|c|c|c|c|c|}
\hline & Rangos & $\mathrm{N}$ & Significado de rangos & Suma de rangos \\
\hline Post - test seriaciones & Rangos negativos & $0^{a}$ & 0,00 & 0,00 \\
\hline \multirow[t]{3}{*}{ Pre - test seriaciones } & Rangos positivos & $10^{b}$ & 6,50 & 78,00 \\
\hline & Semejanza & $0^{c}$ & & \\
\hline & Total & 10 & & \\
\hline
\end{tabular}

Tabla 5: Análisis de cambios significativos en los juegos de clasificación

\begin{tabular}{|c|c|c|c|c|}
\hline & Rangos & $N$ & Significado de rangos & Suma de rangos \\
\hline Post - test clasificaciones & Rangos negativos & $0^{a}$ & 0,00 & 0,00 \\
\hline \multirow[t]{3}{*}{ Pre - test clasificaciones } & Rangos positivos & $11^{\mathrm{b}}$ & 6,50 & 78,00 \\
\hline & Semejanza & $0^{c}$ & & \\
\hline & Total & 12 & & \\
\hline
\end{tabular}


Tabla 6: Análisis de cambios significativos en los juegos de conservación

\begin{tabular}{|c|c|c|c|c|}
\hline & Rangos & $\mathrm{N}$ & Significado de rangos & Suma de rangos \\
\hline Post - test clasificaciones & Rangos negativos & $0^{a}$ & 0,00 & 0,00 \\
\hline \multirow[t]{3}{*}{ Pre - test clasificaciones } & Rangos positivos & $10^{b}$ & 6,50 & 78,00 \\
\hline & Semejanza & $0^{c}$ & & \\
\hline & Total & 12 & & \\
\hline
\end{tabular}

Como se puede ver en la tabla 5, el número de elementos para los cuales el valor de la variable post-test clasificación es considerablemente mayor que el de la variable pre-test clasificación; por tanto, se rechaza la hipótesis nula de que la valoración de pre-test clasificación es igual que la del post-test clasificación para $5 \%$ de nivel de significación.

Por lo tanto, se puede concluir que los juegos de clasificación, ayudan a incrementar las operaciones concretas en los niños. Como puede verse en la tabla 6, el número de elementos para los cuales el valor de la variable post test de conservación es considerablemente mayor que el de la variable pre-test de conservación. Por lo tanto, se rechaza la hipótesis nula de que la valoración de pre-test de clasificación es igual que la de post-test clasificación para $5 \%$ de nivel de significación.

En conclusión, se puede afirmar que los juegos de conservación, ayudan a incrementar las operaciones concretas en niños.

\section{DISCUSIÓN}

Se estableció que es posible diseñar un programa para mejorar un estadio del desarrollo intelectual, como son las operaciones concretas, a través de su estimulación. Se demostró que el programa experimental de estimulación de los procesos cognoscitivos sí es efectivo para poder mejorar el desarrollo de las operaciones concretas en los niños de educación primaria. Que, los profesores de todos los niveles educativos deben utilizar procedimientos piagetianos para desarrollar los procesos cognoscitivos de sus alumnos, ya que constituyen un medio eficaz para lograr ese objetivo.

Se encontró en todos los casos, que la muestra obtuvo en la evaluación post experimental puntajes significativamente más altos que en la evaluación pre experimental; lo cual comprobó que la variable independiente tuvo efecto sobre la variable dependiente. Por lo tanto podemos afirmar que los niños estimulados con tareas piagetianas logran mejorar su desarrollo intelectual pues, siguiendo a Majluf (1), se ha demostrado que el desarrollo de la inteligencia no es un proceso que ocurre en forma necesaria y automática conforme el sujeto va creciendo y que la escolaridad no asegura tampoco el que los sujetos lleguen a las formas más elevadas de pensamiento, sino de que es muy importante su estimulación. Es decir, como bien manifestó Frisancho (2), si se estimula, se pueden dar avances estructurales en el nivel de razonamiento.

Es fundamental atender al desarrollo del razonamiento lógico matemático, sobre todo durante las primeras etapas del desarrollo intelectual, teniendo en cuenta, la plasticidad del cerebro en estas primeras etapas Ruesga (7). De ahí que sea fundamental el papel que cumplen los juegos lógicos ya que no sólo son medio eficaz, para el desarrollo cognitivo sino que estimulan las capacidades lógico matemáticas, lo cual permite que el niño conozca, comprenda y utilice estos conceptos, amplíe su vocabulario, de forma más creativa y con menor esfuerzo Carrasco (4); asimismo, incrementa la utilización de formas argumentativas en la resolución de problemas, obtiene mayor satisfacción en el trabajo cooperativo y desarrolla su autonomía en la realización de las actividades escolares. Ruíz (8), por otro lado, manifiesta que al trabajar con niños mediante el juego, se prioriza el aprendizaje y no la enseñanza, puesto que la metodología se centra en el estudiante y le brinda la oportunidad de desarrollar, con mayor plenitud, sus capacidades psicogenéticas Macías (5). 


\section{REFERENCIAS BIBLIOGRAFCAS}

1. Majluf A. Marginalidad, Inteligencia y Rendimiento Escolar: Desarrollo del Pensamiento Formal Proposicional y Combinatorio de dos grupos de adolescentes de diferentes estratos socio-económicos de Lima Perú. 1ra edición. Lima: Brandon Enterprise S.R. Ltda.; 1993.

2. Frisancho $\mathrm{S}$. Razonamiento probabilístico en un grupo de estudiantes de una universidad particular de Lima. Perú. Revista de Psicología. 1996; 14(2): 20-22.

3. Meza A, Inga J. Contrastación de dos modelos de entrenamiento operatorio y acumulativo en la inducción de las nociones de conservación. Revista Latinoamericana de Psicología. 1980; 12(2): 30-34.

4. Carrasco S. Juegos lógicos para la comprensión de conceptos y procedimientos matemáticos. Madrid: Morata; 2000.

5. Macías G. Estrategias para inducir el desarrollo del pensamiento formal en alumnos de bachillerato. México: Universidad de Guadalajara; 2001.

6. Tejeda P. El desarrollo del pensamiento formal en adolescentes con Síndrome de Down. Argentina: en Revista Científica de la Universidad Blas Pascal. 7(16); 2002.

7. Ruesga P. Educación del razonamiento lógico matemático en educación infantil. [Tesis para optar el título de Doctor en Filosofía y Ciencias de la Educación]. Barcelona: Universidad de Barcelona; 2005. Se encuentra en http://www.scielo.org.ve/pdf/pdg/v29n1/art06.pdf.

8. Ruíz D. Las Estrategias didácticas en la Construcción de las nociones lógico matemáticas en la Educación Inicial. [I Congreso internacional de lógico matemática en Educación infantil]. Venezuela: Universidad de Los Andes; 2008. Se encuentra en: http://www.scielo.org.ve/pdf/pdg/v29n1/art06.pdf.

9. Condemarín M, Chadwick M, Milicic N. Madurez escolar: Manual de evaluación y desarrollo de las funciones básicas para el aprendizaje escolar. Santiago de Chile: Andrés Bello; 1990.

Correo electrónico:

caliaga@continental.edu.pe 